\title{
Attentional effects on concurrent psychophysical discriminations: Investigations of a sample-size model
}

\author{
ANNE-MARIE BONNEL \\ Centre National de la Recherche Scientifique, Marseille, France \\ and \\ JEFF MILLER \\ University of California, San Diego, La Jolla, California
}

\begin{abstract}
In two experiments, a concurrent discrimination paradigm was used to study the effects of visual attention on psychophysical judgments and the consistency of these effects with a sample-size model in which attention influences the variance of the internal representation used to make psychophysical judgments. Two pairs of lines were presented simultaneously-one on each side of fixation-and subjects had to indicate for each pair separately whether or not the lines had the same length. Attention was manipulated by instructing subjects to pay $100 \%, 75 \%, 50 \%, 25 \%$, or $0 \%$ of their attention to the discrimination on one side, with the complementary amount of attention to the other side. In the first experiment, the relationship between attention and discrimination accuracy was consistent with the sample-size model both when attentional allocation varied from trial to trial and when it varied between blocks, and the relationship held over more widely varying attentional allocations than had previously been studied. In addition, discriminations were more accurate overall with varied than with blocked attentional allocation, suggesting that the two types of allocation do not merely differ in the degree to which attention is focused. The second experiment examined the effects of attentional allocation and stimulus variance, the latter being manipulated by randomly incrementing or decrementing line lengths. These manipulations had additive effects on total Thurstonian variance, and a version of the samplesize model gave an excellent quantitative fit to the obtained results. Besides supporting the samplesize model, the results of Experiment 2 suggest that criterion variance is at least as large as sensory variance and that criterion but not sensory variance increases with stimulus variance.
\end{abstract}

The dominant theoretical approach to understanding performance in psychophysical tasks involves a class of models suggested by Thurstone (1927; cf. Luce, 1977; Treisman, 1987). Thurstonian models, of which signal detection theory (SDT) is one example (see, e.g., Green $\&$ Swets, 1966), distinguish two stages of processing. The first is a coding stage, responsible for the translation of a stimulus into a one-dimensional random variable representing its psychological effect. The second is a decision stage, in which a stimulus is categorized by comparing the value of the random variable it elicits against criteria

\footnotetext{
The authors thank Gregory Lockhead, Duncan Luce, George Sperling, and Michel Treisman for their helpful suggestions concerning the research, and Rolf Ulrich for supplying the Rosenbrock function minimization algorithm. F. Gregory Ashby, Rolf Ulrich, Thomas $\mathrm{O}$. Wickens, and two anonymous reviewers provided very useful comments on earlier versions of the manuscript. Requests for reprints should be addressed to A.-M. Bonnel, C.N.R.S.-L.N.F. 1, 31, Chemin JosephAiguier, 13402 Marseille, France, or J. Miller, Department of Psychology, UCSD, La Jolla, CA 92093-0109.
}

-Accepted by previous editor, Charles W. Eriksen defining the boundaries of the different stimulus/response categories.

A major virtue of Thurstonian models is that they allow researchers to incorporate into a single model many disparate factors that affect the detection and recognition of signals embedded in noise. Moreover, these models allow researchers to distinguish and quantify a factor's separate effects on coding ("sensitivity effects") and on decision criteria ("bias effects"). Therefore, Thurstonian models have been used to study the effects of physical stimulus factors (e.g., intensity) which are typically sensory, as well as the effects of more cognitive variables (e.g., payoffs), which generally operate at the decision stage (Green \& Swets, 1966).

This article is concerned with the effects of attention on psychophysical discrimination. Although it is a cognitive variable, attention may nonetheless influence the accuracy with which sensory information is coded (e.g., Downing, 1988; Müller \& Findlay, 1987), and a precise description of this influence in terms of Thurstonian models would both advance our understanding of the relation between attention and accuracy and extend the generality of these models. The character of attentional 
effects is also of major interest as an indication of the nature of top-down control over perceptual processing.

One way to study the relationship between attention and discrimination accuracy is to have subjects perform two concurrent psychophysical discriminations, manipulating (e.g., by instruction) the attention paid to each task. For example, subjects may be instructed to pay a certain proportion of their attention $P(i)$ to discrimination $i$ and the remainder of their attention $[1-P(i)]$ to the other discrimination. Experiments using this technique reveal a performance tradeoff: when the accuracy of one discrimination increases as a result of the allocation of more attention, the accuracy of the other discrimination decreases (see, e.g., Bonnel, Possamai, \& Schmitt, 1987). This tradeoff is typically displayed with the use of a performance operating characteristic (POC) - a plot in which each axis corresponds to performance on one discrimination task and each point displays the joint performance levels on two tasks with a given distribution of attention (see, e.g., Hoffman \& Nelson, 1981; Kinchla, 1969, 1980; Navon \& Gopher, 1979; Norman \& Bobrow, 1975; Sperling \& Melchner, 1978a, 1978b).

\section{OVERVIEW OF PRESENT EXPERIMENTS}

In this article, we report two experiments in which we investigated the ability of a sample-size model, described in detail below, to account quantitatively for performance tradeoffs observed when attention is shared between psychophysical discriminations. We used the line length discrimination paradigm of Bonnel et al. (1987). On each trial, subjects were presented with a pair of vertical lines on each side of fixation. The inner lines were standards, having a fixed length, and the outer lines had the same length or were longer. Subjects first made two comparison responses on each trial, indicating for each pair whether the two lines were same or different in length. ${ }^{1}$ Then, subjects made two confidence judgments, indicating whether they had "high," "medium," or "low" confidence about each of the two "same" versus "different" judgments. In the previous study done with this paradigm, the accuracy of judgments about the lines on a given side was an increasing function of the amount of attention paid to that side, increasing from just above chance to near perfection as the proportion of attention increased from 0.2 to 0.8 . Furthermore, sensitivity (measured by $d_{e}^{\prime 2}$, described below) was an almost perfectly linear function of the proportion of attention paid to a given side, consistent with the sample size model.

The present experiments were designed to evaluate the sample-size model further. In the first experiment, two questions were examined. One was whether the model fits as well when attention is varied from trial to trial (i.e., with the use of cues) as when it is varied between blocks. The sample-size model would have no ready explanation for different patterns of attentional effects in the two conditions, although a substantial change is predicted by certain alternative attentional models (e.g., some all-or-none switching models). The second question was whether the sample-size model could be extended to conditions approximating focused attention. Thus, subjects were told to give $100 \%$ of their attention to the task on one side and $0 \%$ of their attention to the task on the other side. The sample model is consistent with the results from these conditions, although it appears that subjects did not quite succeed in dividing their attention in the all-or-none fashion requested by the instructions.

The second experiment was an investigation of the joint effects of attention and stimulus variance on discrimination accuracy. Stimulus variance was manipulated by adding independent random increments or decrements to the lengths of the two lines being compared. For example, the difference in length between two different lines was not constant across trials, but varied according to a normal probability distribution, the variance of which was manipulated across conditions.

The joint manipulation of attention and stimulus variance provided a relatively strong test of the sample-size model, because, according to this model, both manipulations affect the variance of the internal representations used in making psychophysical judgments. Decreased attention increases representational variance, because it increases the noise inherent in sensory coding; increased stimulus variance also increases representational variance, because it increases the variation in the real-world stimuli being represented. Thus, according to the model, the effects of decreased attention and increased stimulus variance should be comparable, and the model would be supported to the extent that it could quantitatively fit the effects of these analogous manipulations. In fact, when responses were pooled across subjects, discrimination accuracy was fit well by the model.

The results of Experiment 2 also had interesting implications regarding the two components of variance in Thurstonian models: sensory variance (i.e., variation in the internal representation resulting from the presentation of a given stimulus) and criterion variance (i.e., variation in the criteria for deciding which response to make). For one thing, the parameters of the best-fitting model suggested that criterion variance was at least as large as sensory variance. Although variability in the parameter estimates prevented us from concluding this with certainty, the effect was quite apparent in the parameter estimates for 3 of the 4 subjects. More importantly, there were indications that the addition of stimulus variance increased the total variance of the Thurstonian model (henceforth referred to as Thurstonian variance), primarily by increasing criterion variance rather than sensory variance. This finding clearly indicates the importance of the oft-neglected criterion variance in Thurstonian models and provides a new source of support for models in which criterion settings depend on observed stimuli (e.g., Treisman, 1987).

\section{THE SAMPLE-SIZE MODEL}

We begin with a formal specification of the sample-size model, which was adapted to the same-different task by 
Bonnel et al. (1987) from a more general framework suggested by Luce and Green (1978). First, we will describe the basic Thurstonian model for the same-different task as it has been used in focused attention experiments (e.g., Dickman \& Meyer, 1988; Egeth \& Blecker, 1971; Moss, Myers, \& Filmore, 1970). Second, we will indicate how the model is extended to the situation with attention divided across concurrent discrimination tasks. With respect to the modeling of attention, the key assertion is that an increase in attention reduces the variance of the representation extracted by the sensory coding stage.

In the line length comparison task, sensory processing of each line is assumed to produce an internal representation of its length on a unidimensional axis. The location of each line's representation on this axis is probabilistically determined, and, to anticipate the results slightly for the sake of a more concrete specification of the model, there is evidence that these probability distributions are approximately normal and have approximately equal variances, $\sigma_{s}^{2}$.

On each trial, the subject bases the response on the difference in the internal representations. ${ }^{2}$ Given that the random variables are independent, as is commonly assumed, this difference has a normal distribution with mean $\mu$ and variance $2 \sigma_{s}^{2}$. The observer is assumed to partition the internal decision axis (i.e., the difference axis) into intervals corresponding to the available responses (e.g., Green \& Swets, 1966). In the present experiments, for example, subjects had six response categories (same vs. different, with three levels of confidence for each). Given that the inner line was a standard and the outer line had the same length or was longer, the subject was assumed to maintain five decision criteria dividing the difference axis into six regions corresponding to these response categories. A given response is then selected by determining where the current difference falls in relation to the five decision criteria. ${ }^{3}$

Although criterion variance is often ignored by SDT models because in many paradigms it cannot be estimated separately from sensory variance, there is good reason to believe that decision criteria actually fluctuate from trial to trial as is allowed in more general Thurstonian models (Durlach \& Braida, 1969; Gravetter \& Lockhead, 1973; Treisman, 1987; Treisman \& Williams, 1984). For example, as is assumed by range theory for absolute identification experiments (Durlach \& Braida, 1969; Gravetter \& Lockhead, 1973), it is unlikely that observers will be able to make perfectly consistent judgments on all trials, and "measurement errors"' are unavoidable unless the range over which decisions must vary is very small. Furthermore, as is assumed by criterion setting theory (e.g., Treisman \& Williams, 1984), criterion variance may result from a rational response that favors maximum information transmission. Thus, it seems reasonable to assume that the criteria for different response categories vary from trial to trial. Various results (see, e.g., Ingleby, 1968; Kornbrot, 1980) support the simplifying assumptions that criteria fluctuate approximately normally and that different criteria have equal variance, which we shall denote $\sigma_{c}^{2}$.

Assuming that criterion variation is independent of the variation introduced by sensory coding, the total variance at the decision process is

$$
\sigma_{t}^{2}=2 \sigma_{s}^{2}+\sigma_{c}^{2}
$$

for the same-different judgments under consideration. Many of the predictions tested in the present article concern the effects of experimental manipulations on total variance, $\sigma_{t}^{2}$, so we will generally specify models in terms of their predicted values of $\sigma_{t}^{2}$.

Within Thurstonian models, a very common measure of sensitivity is

$$
d^{\prime}=\frac{\mu}{\sigma_{t}}
$$

Given an assumed value for $\mu$ (e.g., constant across conditions), $\sigma_{t}^{2}$ may be estimated by $\mu^{2} / d^{\prime 2}$, as can be seen by rearranging Equation 2 . Note that $\sigma_{t}$ is inversely related to sensitivity, so predictions about $\sigma_{t}^{2}$ can easily be transformed back into predictions about $d^{\prime}$ by using Equation 2.

The Thurstonian framework also provides very powerful techniques for the analysis of confidence rating data (see, e.g., Green \& Swets, 1966). It is possible to generate a receiver operating characteristic (ROC) curve, which is a curve showing the percentages of hits ("different" responses to different stimuli) and false alarms ("different" responses to same stimuli) that would be obtained as the subject varied his criterion for the "same" response. The assumptions of normal distributions for the internal representations and decision criteria imply that the ROC curve should be linear when plotted on normal probability axes, so gross violations of these assumptions can be detected. Furthermore, the slope of the linear ROC curve is equal to the ratio of the variances of the internal representations of same and different pairs, so the relative variances can be estimated. Finally, it is possible to estimate perceptual sensitivity, $d^{\prime}$, and the locations of the five response criteria that separate the six response categories.

The sample size model extends the Thurstonian samedifferent model to the concurrent discrimination task. In essence, attention is defined as a function that controls the amount of sensory information on which a decision is based, the quality of the sensory code being modulated by the number of samples taken from each stimulus. There are two main assumptions.

The first assumption is that the internal representation of each stimulus line is formed by taking a number, $N(i)$, of independent samples of that line, and that the values of these different samples are averaged in order to get a single representation of the length of a line. Predictions of this averaging rule have been confirmed in several experiments by explicitly manipulating the number of stimulus presentations given to the subject or the time preceding a decision (see, e.g., Nosofsky, 1983; Swets \& 
Birdsall, 1967; Swets, Shipley, McKey, \& Green, 1959), and it seems reasonable that the same combination rule would be used for multiple samples taken from a single presentation.

The second assumption is that a fixed total perceptual capacity (i.e., number of perceptual samples) is distributed among concurrent tasks approximately in accordance with the instructions (e.g., approximately $75 \%$ of samples are allocated to a side when the instructions indicate that $75 \%$ of attention should be paid to that side). The subject is further assumed to divide perceptual samples equally between the two lines associated with a given task (i.e., left vs. right of fixation), because this would be optimal. For convenience, we use $2 N$ to denote the total number of samples that can be taken, and $N(i)$ to denote the actual number of samples allocated to each line of the pair on side $i$ [i.e., $2 N(i)$ samples are equally divided between the pair of lines on side $i, i=$ left or right]. Because total perceptual capacity is fixed at $2 N, 2 N($ left $)+$ $2 N$ (right) must equal $2 N$ on each trial.

The main consequence of these assumptions is that the variance of the sensory representation of the length of a given line is inversely proportional to the amount of attention (i.e., number of samples) allocated to it. Let $\sigma_{s}^{2}$ be the variance of the internal representation of a line's length in the focused attention condition (i.e., $N$ samples allocated to each of the two lines to be discriminated). When there are only $N(i)$ samples per line, then, the variance of the representation of each line length will be

$$
\sigma_{s}^{2}(i)=\sigma_{s}^{2} \frac{N}{N(i)}=\frac{\sigma_{s}^{2}}{A(i)},
$$

where $A(i)$ is simply the ratio of the actual number of samples per line to the total possible: $N(i) / N$, which can be regarded as the proportion of attention allocated to side $i$. The variance of the difference in line lengths, on which the same versus different decision is based, is thus $2 \times \sigma_{s}^{2} / A(i)$.

The sample-size model makes specific quantitative predictions about the accuracy tradeoff for responses in two concurrent tasks, $d^{\prime}$ (left) and $d^{\prime}$ (right). The accuracy of responses in a task, $i$, given $N(i)$ samples and a mean difference of $\mu(i)$, is

$$
d^{\prime}(i)=\frac{\mu(i)}{\sqrt{\frac{2 \sigma_{s}^{2}}{A(i)}+\sigma_{c}^{2}}},
$$

assuming that $\sigma_{s}^{2}$ and $\sigma_{c}^{2}$ are the same for the left and right tasks. As noted by Bonnel et al. (1987), if $\mu$ (left) equals $\mu$ (right), as would be expected with equal line length differences on the two sides, and if $\sigma_{c}^{2}$ equals zero (i.e., no criterion variance), then however the fixed total sample size is distributed between the two sides, it follows from Equation 4 that

$$
d^{\prime 2} \text { (left) }+d^{\prime 2} \text { (right) }=\text { constant. }
$$

Thus, under these conditions, the sample-size model predicts that the POC relating $d^{\prime}$ (left) to $d^{\prime}$ (right) will be a segment of a circle. Furthermore, if subjects allocate samples exactly in accordance with the instructed proportion of attention [i.e., if $A(i)=P(i)$ ], then $d^{\prime}(i)^{2}$ will be directly proportional to the instructed proportion of attention (cf. Lindsay, Taylor, \& Forbes, 1968; Taylor, Lindsay, \& Forbes, 1967),

$$
d^{\prime}(i)^{2}=d^{2} \cdot P(i), 0 \leq P(i) \leq 1,
$$

where $d^{\prime}$ is sensitivity in a task when it is given full attention. Both of these predictions were confirmed, for concurrent line length discrimination tasks, under the conditions examined by Bonnel et al. (1987). Thus, the earlier results are consistent with the sample-size model and the additional assumption that $\sigma_{c}^{2}$ is zero. ${ }^{4}$

\section{EXPERIMENT 1}

One reason for performing Experiment 1 was to test the sample-size model under conditions of more extreme attentional imbalance. Bonnel et al. (1987) examined conditions in which subjects were asked to devote $20 \%, 50 \%$, or $80 \%$ of their attention to one side, and the resulting performance tradeoff was well fit by Equation 5. The range of attentional variation was sufficiently small, however, so that the tradeoff was approximately equally well described by a straight line (see Figure 3 of Bonnel et al., 1987). Therefore, this range was extended in the present experiment, and subjects were sometimes asked to devote $100 \%$ of their attention to one task and $0 \%$ to the other. The wider range of variation produced by more extreme attentional allocations should provide a more stringent test of the curvilinear POC predicted by the sample-size model.

A second reason for performing this experiment was to see whether the sample-size model would fit as well when attentional allocation varied from trial to trial as when it varied from block to block. To vary attentional allocation, a cue was presented at the beginning of each trial to indicate the relative priority of the tasks on that trial. Except for the trial-to-trial variation in attention instructions, the varied allocation condition was as close as possible to the conditions used by Bonnel et al. (1987). As described above, the sample-size model involves a mechanism that truely shares attention, because perceptual samples are divided within a trial according to instructed priority. This mechanism should operate similarly whether attention is reallocated from block to block or trial to trial. Thus, one motivation for examining varied allocation is to extend the generality of the sample-size model.

An additional motivation for comparing varied and blocked allocations was to decide between two possible explanations for differences that have previously been observed between these two conditions in cuing paradigms. In focused attention experiments, attention has been found to be focused better when the locus of attention varies from trial to trial than when it is constant (see, e.g., Posner \& Cohen, 1984; Posner, Cohen, Choate, Hockey, \& Maylor, 1984; Posner, Snyder, \& Davidson, 1980), although the effect has not always been obtained (see, e.g., Hillyard, Munte, \& Neville, 1985; Miller, 1991). 
One explanation for this varied cuing advantage is that active processing is required to direct attention to a given region of visual space, and subjects do not or cannot continue this active processing unless there is variation in the location to which they must direct attention (see, e.g., Spencer, Lambert, \& Hockey, 1988). In terms of the sample-size model, this explanation amounts to the claim that subjects in the blocked condition do not focus their attention sufficiently, allocating too much attention (i.e., too many samples) to the low-priority side and not enough to the high-priority side. Note, for example, that this explanation predicts equal performance in the two conditions when $50 \%$ of attention is assigned to each task, because with these instructions attention would not be focused in either of the two conditions. Even with unequal allocation, according to this explanation, the varied and blocked conditions ought to produce points on the same POC, because these conditions differ only with respect to the allocation of samples, not the total sample size. Thus, the only difference in the POCs should be that the varied one should demonstrate more extensive tradeoffs (i.e., larger changes in $d^{\prime}$ as a function of attention) than the blocked one.

A different explanation of the advantage for varied allocation is that it leads to more effective perceptual processing than does blocked allocation. In terms of the sample-size model, more efficient perceptual processing would imply more total samples, so the POC obtained with varied allocation should reflect better overall performance than the POC obtained with blocked assignments (e.g., an advantage in the $50 \% / 50 \%$ condition). Such differential overall performance would rule out the previous hypothesis that the advantage for varied over blocked allocation results simply from insufficiently focused attention.

\section{Method}

Apparatus and Stimuli. Subjects were tested in a sound-shielded cubicle, with dim lighting arranged to be as homogeneous as possible. A PDP-12 (Digital Equipment Corporation) computer was programmed to present the stimuli on a cathode-ray tube (CRT) and to record responses and their latencies. The CRT was coated with P31 phosphor, which decays to $1 \%$ in $250 \mu$-sec, and the brightness of the screen was reduced to minimize persistence. A chinrest was used to keep the distance between the subject's eyes and the CRT constant at $0.65 \mathrm{~m}$.

The stimuli were two pairs of vertical lines presented simultaneously, for $50 \mathrm{msec}$, to the left and right of fixation. The inner line of each pair always subtended $6.21^{\circ}$ of visual angle. The outer line had the same length on half of the trials, and it subtended $6.56^{\circ}$ of visual angle on the other half. The inner and outer lines were presented $2.8^{\circ}$ and $3.3^{\circ}$ from fixation, respectively.

Subjects responded to stimuli on the left and right of fixation by using two five-key response panels positioned under the left and right hands, respectively. For each side, the key under the thumb was used to indicate that the two lines had the same length, and the key under the little finger was used to indicate that they had different lengths. High, medium, or low confidence in each response was indicated by pressing the key under the index, middle, or ring finger, respectively.

Subjects and Procedure. Six of the subjects tested by Bonnel et al. (1987) were called back for further testing, and there were in addition 11 new subjects from the same population. All were paid 35 French francs per hour for participation, and all reported having normal or corrected-to-normal vision. None was aware of the purpose of the study.

Each of the 11 new subjects was tested with varied attentional allocation over the course of three identical 2-h sessions, the first of which was devoted to training (data from this session were not scored). Each session consisted of two blocks of 144 trials, with blocks separated by a few minutes' rest. Each block included 12 trials in each of the 12 conditions resulting from the crossing of the three factors: left stimulus pair (same vs. different), right stimulus pair (same vs. different), and attentional allocation $(20 \% / 80 \%$, $50 \% / 50 \%, 80 \% / 20 \%$ ). The order of trials was randomized separately for each block.

The 6 subjects tested by Bonnel et al. (1987) were retested in a complementary session exploring the limiting case in which the subject was to allocate all his attention to one side while responding to both $(0 \% / 100 \%$ and $100 \% / 0 \%$ instructions). This session included two blocks of 64 trials with attention varied randomly between sides, two blocks of trials with $100 \%$ of attention always devoted to the left side, and two blocks of trials with $100 \%$ of attention always devoted to the right side. The order of the 6 blocks was balanced across subjects.

For all subjects and conditions, each trial began with the onset of one of five cues, lasting $500 \mathrm{msec}$, which indicated the proportion of attention to devote to each task. The " <" and " > " cues instructed subjects to allocate $100 \%$ of their attention to the left and right pairs of lines, respectively. The " $<-$ " and " $->$ " cues instructed subjects to allocate $80 \%$ of their attention to the left and right sides, respectively, with the remaining $20 \%$ allocated to the other side. The " +" cue instructed subjects to allocate $50 \%$ of their attention to each side. To equate brightness, each cue consisted of the same number of illuminated points on the screen.

When it disappeared, the cue was replaced by a fixation point. Subjects were instructed to fixate this point, allocate attention according to the cue, and then press a footswitch when ready for the stimulus display. The stimulus display appeared $150 \mathrm{msec}$ after the footswitch was pressed, and it disappeared $50 \mathrm{msec}$ later. The instructions strongly emphasized the importance of fixating the fixation point before pressing the footswitch, and subjects reported that they could obey these instructions easily, given the small visual angle (about $6^{\circ}$ ) between concurrent stimuli. Since only $200 \mathrm{msec}$ separated the press of the footswitch and the disappearance of the stimuli, it seems unlikely that eye movements were made after pressing the footswitch (see, e.g., Posner, 1980). ${ }^{5}$

After the display disappeared, subjects made two keypress responses to indicate whether they thought each pair of lines had been the same or different in length. Subjects could make the two responses for the two pairs of lines in either order. After both responses had been made, the subjects made two further keypresses to rate their confidence in the responses. The second confidence rating extinguished the fixation point, and a cue appeared to begin the next trial $3 \mathrm{sec}$ later.

Data analysis. Following Bonnel et al. (1987), responses from each of the two concurrent tasks were summarized by using standard procedures to compute ROCs and to estimate parameters of the Thurstonian model from the ROCs. Specifically, the "same" versus "different" responses and confidence judgments were combined to form six distinct response categories, ordered to reflect increasing confidence that the lines were different in length (i.e., from high confidence same to high confidence different). This procedure yielded 5 points on an ROC curve, from which optimal estimates of the parameters of each ROC as well as their variance were obtained by an iterative maximum-likelihood procedure (Dorfman \& Alf, 1969). In order to increase the stability of the estimated parameters of the Thurstonian model for model fitting, responses were generally pooled across subjects before using these procedures, as recommended by Macmillan and Kaplan (1985). In addition to these pooled estimates, we also report the means and standard er- 
Table 1

Experiment 1: Estimated $d_{\mathrm{e}}^{\prime}$ as a Function of Attention, Condition, and Side

\begin{tabular}{|c|c|c|c|c|c|c|c|c|c|c|c|c|}
\hline \multirow[b]{3}{*}{ Attention } & \multicolumn{6}{|c|}{ Blocked } & \multicolumn{6}{|c|}{ Varied } \\
\hline & \multicolumn{3}{|c|}{ Left } & \multicolumn{3}{|c|}{ Right } & \multicolumn{3}{|c|}{ Left } & \multicolumn{3}{|c|}{ Right } \\
\hline & $P$ & $M$ & $S E$ & $P$ & $M$ & $S E$ & $P$ & $M$ & $S E$ & $P$ & $M$ & $\overline{S E}$ \\
\hline $0 \%$ & .80 & 1.11 & .23 & .58 & .86 & .28 & .74 & .99 & .32 & .41 & .93 & .23 \\
\hline $20 \%$ & .75 & .78 & .09 & .79 & .72 & .16 & .71 & .96 & .21 & .85 & .86 & .16 \\
\hline $50 \%$ & 1.32 & 1.11 & .10 & 1.22 & 1.30 & .11 & 1.50 & 1.94 & .26 & 1.45 & 1.69 & .20 \\
\hline $80 \%$ & 1.70 & 1.50 & .14 & 1.69 & 1.62 & .14 & 1.98 & 2.54 & .24 & 2.08 & 2.25 & .28 \\
\hline $100 \%$ & 1.97 & 2.47 & .16 & 1.83 & 2.19 & .21 & 2.33 & 2.77 & .34 & 2.20 & 2.68 & .32 \\
\hline
\end{tabular}

Note-Estimate $P$ was obtained from pooled data, and estimate $M$ was obtained by averaging the estimates obtained from individual subjects. $S E$ is the standard error, across subjects, of the estimate $M$

rors of the estimates computed for individual subjects. For hypothesis tests and model fits, we report results of computations made with both pooled and averaged estimates.

In one set of analyses, responses were pooled across tasks that received the same amount of attention (e.g, left responses from the $80 \% / 20 \%$ condition were pooled with right responses from the $20 \% / 80 \%$ condition). This set of analyses was designed to relate sensitivity to attention allocation (cf. Norman \& Bobrow, 1975). In a second set of analyses, responses for the left and right tasks were analyzed separately, so that the between-task tradeoff function (POC) could be examined.

In each condition, values of total Thurstonian variance, $\sigma_{t}^{2}$, were estimated by using Equation 2 and the observed values of $d_{e}^{\prime}$. The values of $\mu$ were assumed to be equal across tasks, given that the differences were the same size on both sides. These values were arbitrarily set to 11 , the line length difference in pixels on different trials, to facilitate comparison with parameter values estimated in Experiment 2.

One way to evaluate a subject's decision making within the framework of Thurstonian models is to evaluate $\beta$, the ratio of the conditional probability of reporting a difference given the presentation of a different pair, to the conditional probability of reporting a difference given presentation of a same pair. When same and different stimuli have the same a priori probability, the decision criterion should be placed at $\beta=1$ in order to optimize the percentage correct. In order to evaluate response bias, $\beta$ values were estimated for each observer under each experimental condition.

\section{Results and Discussion}

Table 1 shows estimates of $d_{e}^{\prime}$, computed both by pooling responses across subjects and by averaging $d_{e}^{\prime}$ values estimated for individual subjects. Agreement between these estimates is quite good $(r=.94)$, although the average estimates are consistently slightly higher $[M=1.34$ vs. $M=1.56, t(19)=4.04, p<.01$; cf. Swets \& Pickett, 1982].

Figure 1 shows the relationship between sensitivity, measured with the pooled estimates of $d_{e}^{\prime 2}$, and the proportion of attention allocated to a task, $P(i)$. The three points for the blocked conditions with $20 \%, 50 \%$, and $80 \%$ attention are the same data reported by Bonnel et al. (1987); the other data are new. Since the data were collected with the same apparatus and procedures, and since the results were fairly consistent across observers, it seems appropriate to consider the results to be a single, homogeneous data set. As predicted by the sample-size model, a linear model constrained to go through the origin gives a very good account of these data (varied, $r^{2}=.992$; blocked, $r^{2}=.989$ ), and also for the corresponding estimates obtained by averaging rather than pooling (varied, $r^{2}=.986$; blocked, $r^{2}=.902$ ). A slight positive acceleration seems to be present for both blocked and varied conditions, but a quadratic term does not significantly improve model fits for either condition (all $p s>.15$ ). The present results thus extend previous ones by indicating that the sample-size model provides a good quantitative account of the relation between attention and performance even with instructions to allocate attention very extremely $(100 \%$ vs. $0 \%)$, and with attentional allocation varied from trial to trial rather than block to block.

It is also clear from Figure 1 that sensitivity increases more rapidly with attention when allocation is varied rather than blocked, and a statistical comparison of the slopes of the best-fitting lines verified this conclusion. Specifically, the 10 points in the figure are fit significantly better by a two-parameter regression model allowing different slopes in the two conditions than by a one-parameter model requiring the same slope in both conditions $[F(1,8)=26.168, p<.001$; in both models, the lines were constrained to go through the origin]; the same comparison was also significant in the parallel analysis of $d^{\prime 2}$

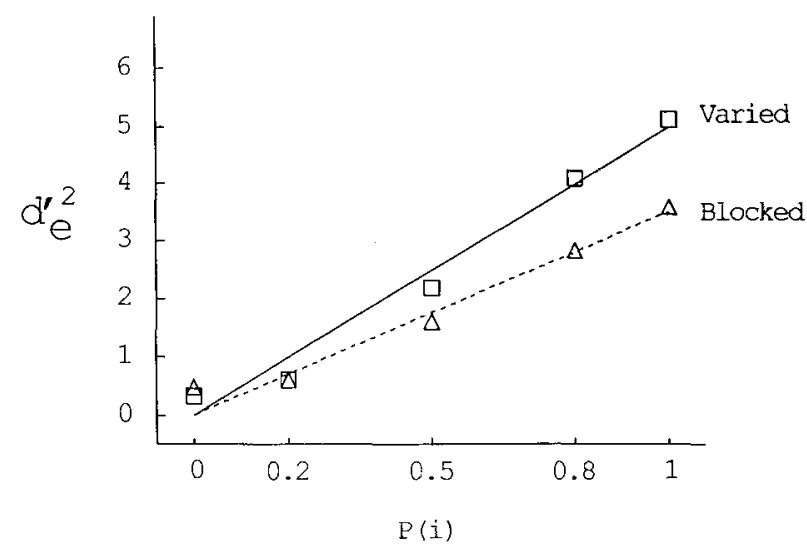

Figure 1. Experiment 1: Sensitivity $\left(d_{e}^{\prime 2}\right)$ as a function of the instructed proportion of attention, $P(i)$, for conditions with blocked and varied attentional instructions. Data for the middle three blocked conditions come from Bonnel et al. (1987). The lines are the bestfitting linear functions constrained to go through the origin. 
values obtained by averaging estimates from individual subjects $[F(1,8)=12.636, p<.01]$. This finding is consistent with the previous evidence that attention may be focused better when the locus of attention varies from trial to trial than when it is constant (e.g., Posner et al., 1980).

Together with the common intercept, the steeper slope in the varied condition than in the blocked one indicates that perceptual processing is more effective with varied allocation. The source of the perceptual advantage for varied allocation is not clear, although it could result from greater inhibition of previously attended locations when allocation is blocked (Posner \& Cohen, 1984). In any case, more efficient perceptual processing would explain the higher slope with varied allocation, because sensitivity would naturally increase faster with the proportion of attention for a more efficient discrimination mechanism.

The results are not consistent with the alternative explanation of the varied-allocation advantage in terms of insufficiently focused attention, which predicted equal performance in the $50 \% / 50 \%$ conditions with the two types of allocation. In essence, the explanation is contradicted by the fact that discriminations are better overall, not simply less influenced by attention, for varied than blocked allocation. This point is also made clearly by the POCs for the two designs (Figure 2), discussed below.

Because the linear growth of $d^{\prime 2}$ with $P(i)$ is consistent with the sample-size model, it supports the claim that the variance of the internal representation is modulated by the instructed proportion of attention allocated to the task. The finding of linearity with both blocked and varied attentional conditions thus extends the generality of the sample-size model.

It is necessary to consider whether alternative models of attentional allocation might also be consistent with the present results. One such model is the attentional switching model (cf. Jonides, 1980, 1983; Kinchla, 1969, 1980; Sperling \& Melchner, 1978b), according to which subjects attend exclusively to one side on any given trial. To allocate attention in accordance with the instructed proportions, subjects vary the proportion of trials on which they attend to each side, thus giving each side its instructed priority on the average across trials.

There are at least two arguments against an account of the present data in terms of such probabilistic switching. First, varying attentional instructions from trial to trial might make it difficult for subjects to implement a switching strategy. If subjects using such a strategy kept count of the number of trials on which they attended exclusively to each side, the complexity of keeping such counts would be greatly increased when the different attentional conditions were mixed together. In that case, attentional effects would probably be different in the blocked and varied conditions.

Second, the switching model is difficult to reconcile with the results of a correlation analysis suggested by Sperling (1984). If subjects switch attention back and forth between tasks from trial to trial, the accuracy of responses on one task should be negatively correlated (across trials) with the accuracy of responses on the other task. In essence, when one task has the all-or-none attentional mechanism, it should be performed well but the other task should be performed poorly. However, trial-by-trial analysis of joint performance indicated that response accuracies (i.e., correct vs. erroneous "same" vs. "different" responses on the two tasks) were virtually independent. The analysis pooling across subjects yielded phi correlation coefficients of $.062, .016,-.026, .030$, and -.030 in the conditions with $0 \%, 20 \%, 50 \%, 80 \%$, and $100 \%$ of attention to the left side, respectively. Phi correlations computed for each subject and attentional condition separately were also near zero, with over half in the interval $(-.09, .09)$, and the remainder about equally divided between the intervals of $(-.19,-.10)$ and $(.10, .19){ }^{6}$

It should be noted that the lack of a negative correlation is compatible with the sample-size model, because this model asserts that attention is truly shared between tasks. Even though the two tasks compete for attentional resources, sharing allows the amount of resources allocated to a given task to remain constant across trials (i.e., it is not necessary to create a given average allocation by a probability mixture of greater and lesser allocations). If resource allocation is constant across trials, trial-to-trial fluctuations in task performance would not result from changing attentional allocations, and performance on the two tasks would be uncorrelated. Thus, the lack of negative correlation may be interpreted in terms of the samplesize model as evidence that there is little or no trial-totrial fluctuation in the proportion of samples allocated to each task. If there were such fluctuation, it would produce a negative correlation, because allocations to the two tasks are complementary: when one task received an especially high proportion (increasing sensitivity), the other task would necessarily receive an especially low proportion (decreasing sensitivity).

In sum, the difficulty of accounting for the present data with a switching model strongly suggests that the dual task is performed with attention truly distributed across tasks (cf. Eriksen \& St. James, 1986). The effects of attentional instructions further indicate that all locations need not receive the same degree of processing within such a distributed processing mode. Taken together, these patterns of data support the sample-size attention model in which a fixed number of observations (i.e., capacity) are apportioned between locations roughly in accordance with attentional instructions.

One observation that appears to raise problems for the sample-size model is that accuracy in the conditions with $0 \%$ attention was significantly better than the value of zero expected for chance performance with both varied $\left(d_{e}^{\prime}=\right.$ $0.56 ; z=8.00, p<.0001)$ and blocked $\left(d_{e}^{\prime}=0.68 ; z=\right.$ $13.6, p<.0001$ ) allocation of attention. ${ }^{7}$ If subjects completely ignored one of the presented pairs as requested, the sample-size model would predict chance performance, because there would be no perceptual information on which to base a decision. The result with pooled data is not due to a small number of subjects who discriminated 
well in the $0 \%$ condition, because every subject had $d_{e}^{\prime} s$ greater than zero in the $0 \%$ conditions with both blocked and varied allocation, and average values of $d_{e}^{\prime}$ were significantly different from zero by $t$ tests [varied, $M=$ $.958, t(5)=3.815, p<.02$; blocked, $M=.987, t(5)=$ $3.945, p<.02$ ]

One explanation of the better-than-chance performance with $0 \%$ attention is that some information is extracted from a stimulus even if no perceptual samples are taken from it, contrary to the sample-size model. Another explanation, also inconsistent with the sample-size model, is that asymptotic performance in the $100 \%$ condition does not require full attentional capacity, so the spare capacity is allocated to the other $(0 \%)$ task. Fortunately, there is also an explanation consistent with the sample-size model; namely, that the instructions to ignore one of the presented pairs were not completely obeyed, possibly because the subject cannot completely control the assignment of perceptual samples. Indeed, subjects confessed that they had been unable to ignore one side completely when they knew that a response was required to stimuli on that side. This explanation is also consistent with the fact that the observed value of $d_{e}^{\prime 2}$ in the $100 \%$ condition falls on the same linear function as do the values of $d_{e}^{\prime 2}$ from the various divided attention conditions. The common functions suggest a common sharing strategy-with only a quantitative difference-across all conditions.

For a more detailed analysis of the performance tradeoff produced by the manipulation of attention, full POCs were obtained by computing separate sensitivity measures for the left and right tasks in each of the different attention conditions, and these are shown in Figure 2. The observed POCs are consistent with the curves predicted by the sample-size model, although the data are clearly not precise enough to rule out alternative relationships. In fact, each of the two five-point POCs is fit slightly better by a linear function (correlations of .99 in both conditions)

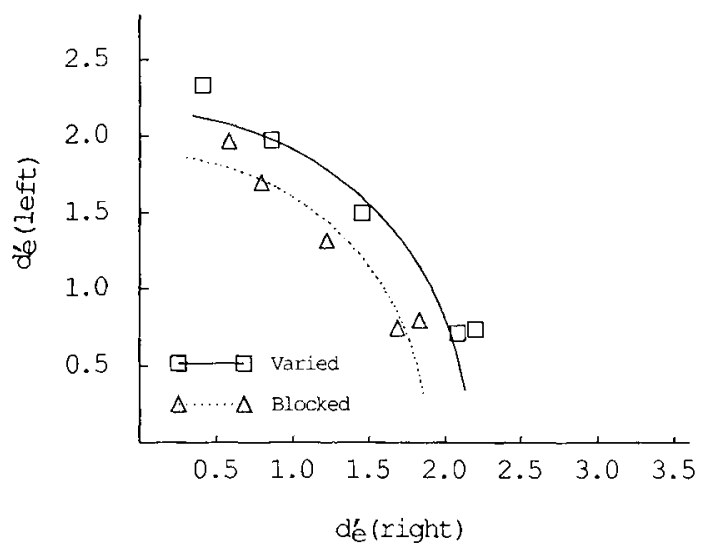

Figure 2. Experiment 1: Performance operating characteristics (POCs), showing d' for each task, with the different points coming from different attentional conditions. The lines show the best fits of the sample-size model to the POCs. than by the sample-size model's curvilinear one (correlations of .96 and .97 for the blocked and varied conditions, respectively). For the varied condition, similar correlations were obtained by using the $d_{e}^{\prime}$ estimates computed by averaging across subjects (linear $r^{2}=-.97$, curvilinear $r^{2}=-.94$ ), but neither model provided a good fit to the highly nonmonotonic results from the blocked condition (linear $r^{2}=-.63$, curvilinear $r^{2}=-.55$ ).

For each type of allocation (varied and blocked) separately, the sample-size model of Equation 4 was fit to the five pairs consisting of one value of $d_{e}^{\prime}(\mathrm{left})$ and one value of $d_{e}^{\prime}$ (right). The model was fit with seven free parameters: $\sigma_{s}^{2}, \sigma_{c}^{2}$, and the values of $A(\mathrm{left})$ for each of the five levels of attention allocation. ${ }^{8}$ The variance parameters were constrained to be non-negative, and the values of $A(i)$ were constrained to the range from 0 to 1 .

As is shown in Figure 2, with pooled sensitivity estimates the sample-size model could not be rejected for either type of allocation $\left[\chi^{2}(3)=4.789\right.$ for varied and 5.637 for blocked, $p>.1$ in both cases], so the model describes these POCs adequately. Estimated values of the parameters were $12.878,0.000,0.104,0.106,0.510$, 0.845 , and 0.967 , respectively, with varied allocation, and $16.960,0.000,0.172,0.161,0.545,0.823$, and 0.914 with blocked allocation. For the varied condition, the fit was also quite good with the use of estimates of $d_{e}^{\prime}$ obtained by averaging $\left[\chi^{2}(3)=1.78, p>.1\right.$; parameter estimates of $8.545,0, .12, .144, .577, .896$, and .889], but the model could not fit the nonmonotonic average values of $d_{e}^{\prime}$ obtained in the blocked condition $\left[\chi^{2}(3)=\right.$ $30.5, p<.01$; parameter estimates of $16.628,0, .191$, $.176, .411, .799$, and .950$]$. Judgment based on the bestfitting parameter values suggests that subjects did not allocate their attention in exactly the instructed proportions, as has already been suggested. In the fits to pooled $d_{e}^{\prime}$ s, for example, the estimated proportions of attention actually paid to a side, $A(i)$, varied from .033 to .172 , even when subjects were instructed to pay $0 \%$ of attention to that side. Thus, it is quite consistent with the model to conclude that the better-than-chance performance with $0 \%$ attention is caused by differences between instructed and actual proportions of attention.

It is also interesting that zero was the best estimate of the parameter $\sigma_{c}^{2}$ in all fits. Clearly, this suggests that subjects' decision criteria do not vary from trial to trial, contrary to various earlier claims (see, e.g., Treisman, 1987). In fact, however, the results of Experiment 2 contradict this conclusion, and suggest instead that the model of Equation 4 is incomplete with regard to $\sigma_{c}^{2}$. In view of results presented later, it appears that criterion variance is reduced by attention just as sensory variance is, rather than being constant across attention allocations as assumed by the model. Thus, we will eventually conclude that the estimate of $\sigma_{s}^{2}$ obtained from this model actually includes criterion as well as sensory variance.

A potential weakness of the sample-size model is that, with both varied and blocked allocation, the conditions with $100 \%$ of attention to the right side seem to create 
discontinuities in the POCs. Note that, as attention to the right side increases from $80 \%$ to $100 \%$, the sensitivity gains for the right side are not compensated by costs on the left side, and the POC becomes horizontal. This suggests that a qualitative change is introduced by the requirement to allocate $100 \%$ of attention to the right side, and the sample-size model has no mechanism to explain such a qualitative change. On the other hand, it is difficult to give much weight to this local deviation from the model, because even with the deviation, the data are not sufficient to reject the model.

If we turn from sensitivity to criterion measures, another point of consistency with the sample-size model is that overall response biases were not influenced by attention. In fact, $\beta$ values were not significantly different from one for any distribution of attention, with either varied or blocked attentional allocation.

In summary, this experiment replicated the linear tradeoff between $d_{e}^{\prime 2}$ and the proportion of attention allocated to a task-predicted by the sample-size modelwith attentional allocation varying from trial to trial. Furthermore, with attentional allocation varied over a wider range than was examined previously (Bonnel et al., 1987), the shape of the POC was still consistent with the samplesize model, although it was again difficult to rule out some alternative models based on this shape. The success of the sample-size model suggests several conclusions in terms of standard theoretical analyses of dual-task performance (e.g., Navon \& Gopher, 1979; Norman \& Bobrow, 1975). First, it is clear that when the tasks have to be performed simultaneously, each one suffers from the presence of the other; were this not true, the POCs shown in Figure 2 would be square. Second, the consistency of the performance tradeoff with the model supports the assumption that a given amount of resources (samples) withdrawn from one task can be fully utilized on the other task. In other words, as is assumed in most divided attention models, it appears that total capacity does not vary with attentional allocation.

\section{EXPERIMENT 2}

In this experiment, variance was added to the physical stimuli to enable the investigation of the effects of stimulus variance on psychophysical judgments and to provide a more stringent test of the sample-size model. On each trial, the lengths of the two stimulus lines were independently chosen from normal distributions with variance $\sigma_{l}^{2}$. On same trials, the two lines came from the same distribution, so the mean difference in lengths was zero; because of the variance, of course, the lengths were not always identical. On different trials, the lines came from distributions with different means; both variances were again $\sigma_{l}^{2}$. Thus, there was also variation in the size of the differences on different trials. In short, subjects were confronted with a probabilistic rather than a deterministic decision problem (cf. Kubovy \& Healy, 1977; Lee \&
Jahnke, 1964). As noted by Ashby and Gott (1988), presenting probabilistic stimuli often provides useful extra information about the nature of the perceptual representations formed by the subject.

Adding variance to the physical stimuli increases the variance of the subject's internal representation of line length (Wickelgren, 1968). According to the sample-size model, so does reducing attention. One goal of the experiment, then, was to find out whether reduced attention and increased physical stimulus variance would have comparable effects. To the extent that they do, and to the extent that the sample-size model provides a good quantitative account of those effects, it raises the plausibility that attention influences the variance of the internal representation, as proposed by the sample-size model. In particular, adding variance to the stimuli can be used to estimate the internal variance contributed to these representations by the observer (cf. Barlow, 1978, 1980; Burgess \& Barlow, 1983), and in effect this enabled us to see whether representational variance was influenced by attention as predicted by the model.

The logic of this investigation is roughly similar to that of the studies of Hawkins, Shafto, and Richardson (1988) and Hughes (1984), who examined the relationship of attention and stimulus intensity. Whereas they sought to find out whether increasing attention is comparable to increasing stimulus luminance, we sought to determine whether increasing attention is comparable to decreasing physical stimulus variance. The equivalence of attention and luminance is suggested by the spotlight metaphor (see Umiltà, 1988 , for a review), whereas the conceptual relation between attention and stimulus variance derives directly from the sample-size model, as elaborated below.

For purposes of the present analyses, stimuli were simply classified as same or different according to the distributions from which they were selected, ignoring their true trial-by-trial lengths, so even a perfect observer would make errors. Because the goal of the experiment was to see how externally added stimulus variance would combine with attention to determine sensitivity, it was logically necessary to produce some variance in the physical stimuli within a condition, thereby making the judgments probabilistic. The subjects did notice the probabilistic nature of the stimuli, but they reported no difficulty in making "same"-"different" judgments as instructed. One reason is that they were given no feedback, so they were never confronted with the fact that the experimenter's classifications of the physical stimuli contained some variance. Another reason is that many small differences could not be confidently perceived, especially with brief stimulus displays. Thus, the proportion of apparently same stimulus pairs was much nearer to $50 \%$ than the actual proportion of physically identical pairs.

To extend the sample-size model to an experiment with variation in the physical stimuli, it is helpful to develop the equations starting from the individual random variables that are postulated. In the present experiment, on 
each trial the experimenter randomly selects the lengths $l_{1}$ and $l_{2}$ of the two stimulus lines, with independent selections from normal distributions with $\sigma_{l}^{2} \equiv \operatorname{Var}\left[l_{i}\right]$.

When these lines are presented, the subject forms sensory representations $l_{1}+s_{1}$ and $l_{2}+s_{2}$ of the lengths of these lines, where $s_{i}$ is a normally distributed random increment with $\mu=0$, and the values of $s_{i}$ are assumed to be independent of each other and of the lines presented on the other side. In keeping with the basic assumptions of the sample-size model (cf. Equation 3), sensory variance decreases as attention increases, so the variance of $s_{i}$ is

$$
\operatorname{Var}\left[s_{i}\right]=\frac{\sigma_{s}^{2}}{A(i)} .
$$

Changes in $\sigma_{l}^{2}$ across experimental conditions are assumed not to alter the accuracy of the subject's sensory coding process (i.e., $\sigma_{s}^{2}$ ).

Given these assumptions, the subject's internal representation of the difference is

$$
\Delta \equiv\left(l_{1}+s_{1}\right)-\left(l_{2}+s_{2}\right),
$$

and the variance of this internal representation, $\sigma_{r}^{2}$, is

$$
\begin{aligned}
\sigma_{r}^{2} & =\left(\sigma_{l}^{2}+\frac{\sigma_{s}^{2}}{A(i)}\right)+\left(\sigma_{l}^{2}+\frac{\sigma_{s}^{2}}{A(i)}\right) \\
& =2 \sigma_{l}^{2}+2 \frac{\sigma_{s}^{2}}{A(i)} .
\end{aligned}
$$

Thus, one consequence of adding stimulus variance is to increase the variance of the subject's representation of line length (cf. Wickelgren, 1968). Note that representational variance results partly from variance in the external stimuli and partly from variance contributed by the sensory coding process, but that only the latter source of variance is affected by attention.

After the representation of the stimulus difference $\Delta$ has been formed, the "same"'-"different" decision is based on the sign of the difference $\Delta-c$, where $c$ is a randomly varying decision criterion independent of $\Delta .{ }^{9}$ Assuming that criterion variance also remains constant as $\sigma_{l}^{2}$ changes, total Thurstonian variance (i.e., the variance of $\Delta-c$ ) is

$$
\begin{aligned}
\sigma_{t}^{2} & =\sigma_{c}^{2}+\sigma_{r}^{2} \\
& =\sigma_{c}^{2}+2 \sigma_{l}^{2}+2 \frac{\sigma_{s}^{2}}{A(i)} .
\end{aligned}
$$

With respect to the experimental manipulations of stimulus variance and attention, then, the model predicts additive effects of stimulus variance and attention. Furthermore, total variance should increase linearly with slope two as a function of stimulus variance.

In summary, the question is whether Equation 10 can quantitatively describe the results of a series of conditions in which $\sigma_{l}$ and the proportion of attention are systematically manipulated. A good fit would support the claim that increasing attention decreases the variance of the internal representation, as is assumed by the sample-size model.

\section{Method}

Except for the differences described below, the apparatus, stimuli, and procedure were the same as in the first experiment. Four subjects who had already served in the previous experiment were tested in the 15 conditions resulting from the crossing of three levels of attentional allocation $(25 \% / 75 \%, 50 \% / 50 \%$, and $75 \% / 25 \%)$ and five levels of stimulus variance. In different sessions, the standard deviations of the line length distributions $\left(\sigma_{l}\right)$ were $1,2,3,4$, or 6 pixels. Data were collected from three sets of sessions over several months. In the first set (Sessions 1-3), attention instructions were $50 \% / 50 \%$ and $\sigma_{l}$ was equal to 2 pixels on the left side and 6 pixels on the right (the $2 / 6$ condition), the reverse $(6 / 2)$, or 4 pixels on each side (4/4). In the second set (Sessions 4-9), the previous values of $\sigma_{l}$ were tested in $75 \% / 25 \%$ and $25 \% / 75 \%$ attention conditions. Finally, the third set of 6 sessions (Sessions 10-15) tested stimulus standard deviations of $\sigma_{l}=1$ on the left and three on the right $(1 / 3)$, and vice versa $(3 / 1)$, crossed with the three attention conditions $(25 \% / 75 \%, 50 \% / 50 \%$, and $75 \% / 25 \%)$.

The first two sets of data were collected over sessions made of two blocks of 96 trials per block with rests between blocks. For practical reasons, the third set (Sessions 10-15) was made of oneblock sessions only. Attention instructions were the same throughout the block of trials, and the order of blocks was balanced, as far as possible, across subjects.

Vertical lines varied in length from trial to trial. Length variance was introduced in each pair of lines in the following way: In a first stage, two Gaussian distributions of length with the same standard deviation were constructed. For the shorter lines, the distribution was centered on a mean value of 196 pixels, and for the longer lines, on a mean value of 222 pixels. Each distribution contained 96 elements. The common standard deviation varied from one condition to another $\left(\sigma_{l}=2,4\right.$, or 6 points).

In a second stage, same and different stimuli were constructed as follows. Same stimuli were constructed by randomly selecting two lines from the same distribution of lengths ( $\mu=196$ pixels). The mean of the resulting distribution of differences was thus zero. On different trials, the inner line was also selected from the distribution centered on 196, but the outer line was selected from the distribution centered on the longer length, or 222 , so that the mean of the distribution of differences was 26 pixels.

In the conditions with $\sigma_{l}=1$ and $\sigma_{l}=3$, lines of different lengths were used in order to shift $d_{e}^{\prime}$ into a new range. The construction of lines was as just described, except with a mean of 209 pixels for the longer line (average difference $=13$ pixels for different pairs).

A problem of measurement units arose in this experiment. The parameters of the Thurstonian model refer to the internal scale for the representation of line length, so they must be measured in units appropriate to this scale. This problem does not often arise in psychophysical experiments; the units need not be specified, because it is possible to measure all parameters relative to the single unknown value of $\mu$, which can be set arbitrarily to one. Such an approach could not be used in the present experiment, however. There were two different values of $\mu$, corresponding to mean differences of 13 and 26 pixels, and the values of $\sigma_{l}$ had to be specified in pixels, because their values were unknown in units of the internal scale.

To proceed in spite of this problem, we assumed that the internal representation of line length was linearly related to true length. It seemed likely that this assumption was not too far wrong over the relatively narrow range of line length variation included in this experiment, because magnitude estimates of line length are very nearly a linear function of true physical length (e.g., Stevens \& Galanter, 1957; Teghtsoonian \& Teghtsoonian, 1971). Although a direct inference of the psychophysical scale from magnitude estimates ignores the process of transforming the psychophysical response into 
Table 2

Experiment 2: Estimated Receiver Operating Characteristic Parameters as a Function of Attention and Stimulus Variance $\left(\sigma_{l}\right)$

\begin{tabular}{|c|c|c|c|c|c|c|c|c|c|}
\hline \multirow[b]{3}{*}{$\sigma_{l}$} & \multicolumn{9}{|c|}{ Percentage of Attention } \\
\hline & \multicolumn{3}{|c|}{$25 \%$} & \multicolumn{3}{|c|}{$50 \%$} & \multicolumn{3}{|c|}{$75 \%$} \\
\hline & $P$ & $M$ & $S E$ & $P$ & $M$ & $S E$ & $P$ & $M$ & $S E$ \\
\hline \multicolumn{10}{|c|}{ Estimates of $d_{e}^{\prime}$} \\
\hline 1 & 1.32 & 1.41 & .16 & 1.67 & 1.41 & .21 & 1.97 & 1.88 & .47 \\
\hline 2 & 2.32 & 2.38 & .16 & 2.93 & 2.95 & .23 & 3.22 & 3.15 & .17 \\
\hline 3 & 1.05 & .95 & .07 & 1.30 & 1.26 & .19 & 1.58 & 1.55 & .34 \\
\hline 4 & 1.95 & 2.15 & .09 & 2.17 & 2.20 & .17 & 2.31 & 2.25 & .14 \\
\hline 6 & 1.56 & 1.55 & .26 & 1.62 & 1.66 & .04 & 1.74 & 1.84 & .10 \\
\hline \multicolumn{10}{|c|}{ Estimates of $\beta$} \\
\hline 1 & 1.24 & 1.08 & .24 & 1.13 & 1.59 & .75 & .91 & 1.44 & .80 \\
\hline 2 & .47 & .58 & .15 & .23 & .53 & .35 & .59 & 1.60 & .65 \\
\hline 3 & 1.18 & 1.75 & .69 & 1.06 & 1.29 & .34 & .89 & 1.48 & .55 \\
\hline 4 & .91 & 1.06 & .16 & .50 & .74 & .26 & .70 & .72 & .21 \\
\hline 6 & .68 & 1.52 & .35 & .53 & .58 & .13 & .44 & .49 & .17 \\
\hline
\end{tabular}

Note-Estimate $P$ was obtained from pooled data, and estimate $M$ was obtained by averaging the estimates obtained from individual subjects. $S E$ is the standard error, across subjects, of the estimate $M$.

a magnitude estimate (Treisman, 1987), it is more parsimonious to assume a linear transformation than to assume two nonlinear transformations (length to internal representation, and internal representation to the magnitude estimate) that exactly counterbalance each other so as to produce a linear transformation from length to magnitude estimate.

Given the assumption of a linear transformation from the physical length to the internal representation of length, it was possible to measure all of the parameters of the Thurstonian model in pixels, including the experimenter-determined values of $\mu$ and $\sigma_{l}$. Thus, the values of $\sigma_{t}, \sigma_{s}$, and $\sigma_{c}$ estimated in this experiment were all in units of pixels rather than the arbitrary units normally considered. $^{10}$

\section{Results and Discussion}

Table 2 shows estimates of $d_{e}^{\prime}$ and $\beta$ as a function of attentional allocation and stimulus variance. Once again, $d_{e}^{\prime}$ was used as the sensitivity measure, because the slopes of the ROCs indicated that the variance of the signal distribution was slightly greater than that of the noise distribution. The estimates labeled $P$ were obtained by using responses pooled across observers. The estimates labeled $M$ were obtained by computing parameter estimates separately for each individual subject and condition, and then computing the mean of these values across subjects. The standard errors $(S E)$ were computed from the variance across subjects of the latter set of estimates. The estimates computed from pooled responses were used for all subsequent analyses, except where otherwise noted.

The points in Figure 3 indicate observed sensitivity as a function of attention, stimulus variance, and mean difference in line length. All manipulations had the expected effects. Clearly, sensitivity increases with increasing attention and with increasing difference in line length, but it decreases with increasing stimulus variance.

Values of $\sigma_{t}^{2}$ were estimated for each condition, by using Equation 2, the observed $d_{e}^{\prime}$ values, and the fixed mean size of difference ( $\mu=13$ or $\mu=26$ pixels) in each condition. The resulting values are shown in Figure 4 . The total variance shows nicely additive effects of attention and stimulus variance; the pattern is well described by a family of parallel straight lines $\left(r^{2}=.99\right)$. In short, the effects of attention on total variance are impressively regular and independent of the effects of stimulus variance.

However, Equation 10 clearly does not describe the results accurately, because the common slope of the lines relating $\sigma_{t}^{2}$ to $\sigma_{l}^{2}$ is 5.25 , not 2.0 as predicted. Given that the effects of attention on $\sigma_{t}^{2}$ are quite linear, as the model predicted, it appears that there was an error in one or more of the simplifying assumptions made in adapting the model to the manipulation of stimulus variance.

In fact, previous studies provide good reasons to question our simplifying assumptions that $\sigma_{s}^{2}$ and $\sigma_{c}^{2}$ do not change as $\sigma_{l}^{2}$ is manipulated. The most direct evidence

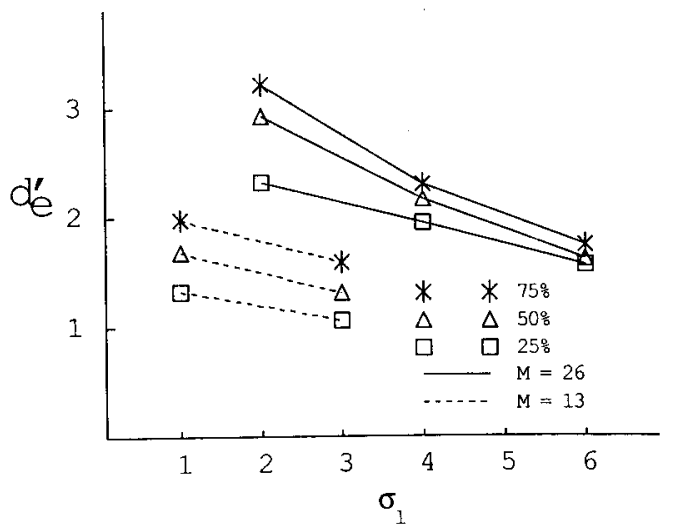

Figure 3. Experiment 2: Sensitivity ( $\left.d_{e}^{\prime}\right)$ as a function of stimulus variability $\left(\sigma_{l}\right)$ for each of the conditions in Experiment 2. The points indicate observed data values, and the lines indicate predicted values obtained from the best-fitting version of the sample-size model (Equation 15). 


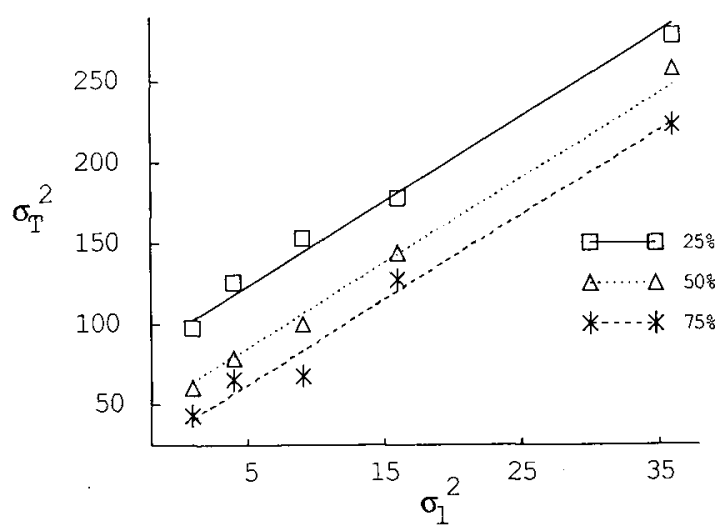

Figure 4. Experiment 2: Estimated total variance $\left(\sigma_{t}^{2}\right)$ as a function of stimulus variance $\left(\sigma_{l}^{2}\right)$. The best-fitting parallel lines are shown in the figure; their slope is $\mathbf{5 . 2 5}$.

that both $\sigma_{s}^{2}$ and $\sigma_{c}^{2}$ would be influenced by changes in $\sigma_{l}^{2}$ comes from Nosofsky (1983), who developed a modelbased technique for estimating sensory and criterion variance in identification tasks. He applied this technique in several conditions differing in the physical range over which stimuli varied, and he found evidence that both sensory and criterion variance increased with stimulus range. In the present experiment, increases in $\sigma_{l}^{2}$ necessarily entail increases in stimulus range, so Nosofsky's results clearly suggest that sensory and criterion variance will increase with $\sigma_{l}^{2}$.

There are also theoretical precedents for the idea that physical stimulus variation will influence sensory and/or criterion variance. According to attention band theory (Luce, Green, \& Weber, 1976), for example, the variance of sensory coding increases with the distance of a stimulus from that which is expected. Obviously, increasing $\sigma_{l}$ increases the average distance between a stimulus and the expected stimulus, so this theory would suggest a corresponding increase in coding variance. ${ }^{11}$ According to range theory (e.g., Braida \& Durlach, 1972; Durlach \& Braida, 1969; Gravetter \& Lockhead, 1973), on the other hand, physical stimulus variation should influence criterion variance but not sensory variance. Criterion-setting theory (e.g., Treisman, 1987; Treisman \& Williams, 1984) also seems to predict a dependence of criterion variance on physical stimulus variation, although it does not directly address the question of whether sensory variance should be affected.

Given the preceding arguments that $\sigma_{s}^{2}$ and $\sigma_{c}^{2}$ may change as $\sigma_{l}^{2}$ is manipulated, it seems appropriate to consider a more general version of the sample-size model than that represented by Equation 10. As suggested by the results of Nosofsky (1983), we assume that sensory variance increases linearly with stimulus variance, in which case Equation 7 becomes

$$
\operatorname{Var}\left[s_{i}\right]=\frac{\sigma_{s}^{2}+k_{s} \sigma_{l}^{2}}{A(i)}
$$

Given this assumption, the variance of the internal representation $\sigma_{r}^{2}$ is

$$
\begin{aligned}
\sigma_{r}^{2} & =\left(\sigma_{l}^{2}+\frac{\sigma_{s}^{2}+k_{s} \sigma_{l}^{2}}{A(i)}\right)+\left(\sigma_{l}^{2}+\frac{\sigma_{s}^{2}+k_{s} \sigma_{l}^{2}}{A(i)}\right) \\
& =2 \sigma_{l}^{2}+2 \frac{\sigma_{s}^{2}}{A(i)}+2 \frac{k_{s} \sigma_{l}^{2}}{A(i)} .
\end{aligned}
$$

As has been noted above, criterion variance may also depend on stimulus variance (cf. Nosofsky, 1983), and the model should be elaborated to allow for this possibility as well. It is tempting to assume that criterion variance increases linearly with stimulus variance, as we did for sensory variance (Equation 11). This assumption seems theoretically incoherent, however. In Thurstonian models, the decision stage has access only to internal representations of the stimuli, not to true physical stimulus values, so that if the decision process were to depend on the stimulus values, it should depend on the encoded values rather than the actual ones. Thus, it seems appropriate to assume that criterion variance may be influenced by all of the representational variance $\left(\sigma_{r}^{2}\right)$ rather than just one stimulus component of it, yielding ${ }^{12}$

$$
\begin{aligned}
\operatorname{Var}[c] & =\sigma_{c}^{2}+k_{c} \sigma_{r}^{2} \\
& =\sigma_{c}^{2}+k_{c}\left[2 \sigma_{l}^{2}+2 \frac{\sigma_{s}^{2}}{A(i)}+2 \frac{k_{s} \sigma_{l}^{2}}{A(i)}\right],
\end{aligned}
$$

where the parameter $k_{c}$ reflects the tendency of $\operatorname{Var}[c]$ to increase linearly with $\sigma_{r}^{2}$.

Based on Equations 12 and 14, total Thurstonian variance (i.e., the variance of $\Delta-c$ ) is

$$
\begin{aligned}
\sigma_{t}^{2} & =\sigma_{r}^{2}+\left(\sigma_{c}^{2}+k_{c} \cdot \sigma_{r}^{2}\right) \\
& =\sigma_{c}^{2}+\left(1+k_{c}\right) \sigma_{r}^{2} \\
& =\sigma_{c}^{2}+2\left(1+k_{c}\right) \sigma_{l}^{2}+2\left(1+k_{c}\right) \frac{\sigma_{s}^{2}}{A(i)}+2\left(1+k_{c}\right) \frac{k_{s} \sigma_{l}^{2}}{A(i)}
\end{aligned}
$$

With respect to the experimental manipulations of stimulus variance and attention, this equation decomposes total variance into a constant $\left(\sigma_{c}^{2}\right)$, a main effect of stimulus variance $\left[2\left(1+k_{c}\right) \sigma_{l}^{2}\right]$, a main effect of attention $\left[2\left(1+k_{c}\right) \sigma_{s}^{2} / A(i)\right]$, and an interaction of stimulus variance with attention $\left[2\left(1+k_{c}\right)\left(k_{s} \sigma_{l}^{2}\right) / A(i)\right]$. Furthermore, if the interaction term can be neglected (i.e., $k_{s} \approx 0$ ), then sensory and criterion variance can be estimated separately, because the former depends on attention but the latter does not. Note also that if sensory and criterion variance are not influenced by stimulus variance (i.e., $k_{s}=k_{c}=0$ ), total variance should increase linearly with slope two as a function of stimulus variance, as in Equation 10. Within this model, deviations from a slope of two can be attributed to an influence of stimulus variance on either sensory or criterion variance, or both.

Is the generalized model instantiated in Equation 15 capable of fitting the data? The model was evaluated formally by fitting it to the full set of $d_{e}^{\prime}$ values shown in 
Table 3

Experiment 2: Parameters, Parameter Estimates, and Bounds for the Sample-Size Model

\begin{tabular}{|c|c|c|c|c|c|c|c|}
\hline \multirow[b]{2}{*}{ Parameter } & \multirow{2}{*}{$\begin{array}{c}\text { Pooled } \\
\text { Estimate }\end{array}$} & \multicolumn{2}{|c|}{ Bounds } & \multicolumn{4}{|c|}{ Estimate for Subject } \\
\hline & & Lower & Upper & 1 & 2 & 3 & 4 \\
\hline$\sigma_{s}^{2}$ & 4.91 & .90 & 9.24 & 1.03 & 1.83 & 17.89 & 0.01 \\
\hline$\sigma_{c}^{2}$ & 10.54 & .00 & 43.30 & 27.49 & 55.48 & 0.0 & 49.68 \\
\hline$k_{s}$ & 0.00 & .00 & 0.22 & 0.0 & 0.0 & 1.06 & 0.0 \\
\hline$k_{c}$ & 1.53 & .74 & 1.82 & 2.07 & 1.05 & 0.0 & 0.94 \\
\hline$A(0.25)$ & 0.27 & .08 & 0.34 & 0.07 & 0.14 & 0.33 & 0.0 \\
\hline$\chi^{2}$ & 2.08 & & & 96.09 & 21.76 & 103.4 & 39.23 \\
\hline
\end{tabular}

Figure 3 (see note 8 ), and the fit was quite acceptable $\left[\chi^{2}(10)=2.077, p>\right.$.99]. There were five free parameters: $\sigma_{s}^{2}, \sigma_{c}^{2}, k_{s}, k_{c}$, and the value of $A(i)$ for the condition with $25 \%$ attention. $A(i)$ was constrained to be 0.50 for the condition with $50 \%$ attention; the conditions are symmetric within this analysis, so the subject cannot be differentially biased. In addition, the $A(i)$ for the $75 \%$ condition is simply one minus that for the $25 \%$ condition. The resulting parameter estimates are shown in Table 3.

The model was also fit to the corresponding individualsubject data values, and the parameter values and goodnessof-fit statistics obtained for the individual subjects are also shown in Table 3. Clearly, the model did not fit nearly as well for individual subjects, but the deviations from the model were quite unsystematic. In fact, every individual subject showed nonmonotonic effects of attention at some values of $\sigma_{l}$ and also showed nonmonotonic effects of $\sigma_{l}$ within some attentional conditions, and we believe that these large deviations from the model reflect random session-to-session variations in motivation and/or sensitivity.

At least for pooled responses, the generalized samplesize model provides an excellent fit to discrimination accuracy as a function of both attention and stimulus variance, but only when sensory and/or criterion variance is allowed to change as a function of experimenter-added stimulus variance. The acceptability of the fit supports the basic tenets of the sample-size model, as well as the incidental assumptions made in the application of the model to this experiment (e.g., that internal representation of length is a linear function of physical length). In particular, the basic idea of the model-that attention serves to reduce sensory noise-is supported by the fact that the effects of external noise (due to added stimulus variance) and internal noise (i.e., due to reduced attention) are both quantitatively describable in terms of changes in total variance within the model.

In view of the fact that the sample-size model adequately fits discrimination accuracies, it is worthwhile to see what conclusions are suggested by the estimated parameter values. To facilitate the interpretation of these estimates, upper and lower $95 \%$ confidence interval bounds were computed for each parameter. To obtain an upper bound, a parameter was fixed at some value, $v$, greater than its optimal estimate, and the model was refit with the other parameters allowed to vary freely. A number of differ- ent values of $v$ were tried, and the upper bound was selected as the smallest value of $v$ for which the fit was significantly worse than the optimal fit with all parameters free to vary (i.e., $\chi^{2}$ increased by 3.84 ). This procedure was easy to implement, because for every parameter the chi-squared values increased monotonically as $v$ deviated from the optimal value. The same procedure was used to obtain a lower bound, except that values of $v$ were of course chosen from below the optimal estimate. Although it is perhaps unusual to obtain confidence intervals by means of an iterative procedure rather than by direct computation, this procedure does provide intervals satisfying the standard statistical criterion: if the procedure were used with an infinite number of samples, the computed interval would contain the true parameter value $95 \%$ of the time (cf. Dudewicz \& Mishra, 1988; Lehmann, 1986), at least to the extent that the computed test statistic is distributed as an approximate chi-square.

It is quite interesting that the best estimate of $k_{s}$ for this model was zero and that the upper bound for $k_{s}$ was relatively small. This indicates that the model fits no better if sensory variance is allowed to change with stimulus variance than if it is assumed to be independent of stimulus variance, and this pattern is apparent in the individualsubject parameter estimates for 3 of the 4 subjects. This conclusion is also supported by the fact that the lines are parallel in Figure 4, because Equation 15 yields additive effects of $\sigma_{l}^{2}$ and $A(i)$ only when $k_{s}=0$.

The conclusion that sensory variance is independent of stimulus variance is inconsistent with that of Nosofsky (1983), whose analysis suggested that changes in stimulus range produced large changes in sensory variance. It is not clear what is responsible for the difference in conclusions, but some obvious candidates are the differences in experimental tasks (i.e., identification vs. discrimination), analyses on which the conclusions were based, and manipulations of stimulus variance versus stimulus range. Although we cannot choose with certainty among the various possibilities, another interpretation of the discrepancy is that one of the assumptions underlying Nosofsky's analysis was not correct in the current task; this point is elaborated on in the General Discussion.

The best-fitting model for the present data suggests that criterion variance, unlike sensory variance, does depend on stimulus variance. This dependence is apparent from the fact that the lower bound for $k_{c}$ was greater than zero, 
both for the pooled responses and for three of the four individual subjects. In fact, the model fit the pooled data significantly worse when $k_{c}$ was constrained to be zero $\left(\chi^{2}=11.49\right.$ vs. $\left.\chi^{2}=2.077, p<.005\right)$, allowing rejection of the null hypothesis that criterion variance is independent of stimulus variance.

The optimal parameter estimates for $\sigma_{c}^{2}$ and $\sigma_{s}^{2}$ suggest that criterion variance is larger than sensory variance (10.54 vs. 4.91 ), but little can be made of these parameter estimates because the fit is very insensitive to the relative values of these parameters, as is shown by their wide and overlapping bounds. In the present design, it is quite difficult to separate the estimates of $\sigma_{s}^{2}$ and $\sigma_{c}^{2}$, because they are strongly negatively correlated with one another (i.e., decreases in one parameter compensate for increases in the other, as is clear from Equation 15). Nonetheless, optimal parameter estimates for 3 of the 4 individual subjects suggest that criterion variance is much greater than sensory variance.

As in Experiment 1, phi correlation coefficients, computed to measure the statistical dependence between the accuracy of left and right responses, were non-negative, contrary to a model in which all-or-none switching accounts for attentional allocation. Correlations computed for each subject separately were all quite close to zero, and the correlations computed by pooling across subjects were uniformly positive, and significantly so $(p<.01)$ in 10 out of 15 conditions. The lack of negative correlations is consistent with the sample-size model, as discussed previously. The positive correlations may reflect slight differences in overall subject sensitivities.

With respect to response bias, neither attention nor stimulus variability induced systematic variations of $\beta$, although there was a general tendency for $\beta$ to decrease below one when variability was large $\left(\sigma_{l}>3\right)$. Some bias toward "different" responses is not surprising, despite the fact that half the stimulus pairs were extracted from the same probability distribution of lengths, because more than half the pairs were noticeably different. In an analysis of variance on $\beta$ s estimated from data of individual subjects, there was no significant effect of attention or $\sigma_{l}$, nor was there an interaction of these two factors (all ps $>.10)$.

\section{GENERAL DISCUSSION}

As in previous studies with the concurrent discriminations paradigm (e.g., Bonnel et al., 1987; Sperling \& Melchner, 1978a, 1978b), the present results indicate that attention has a large effect on the sensitivity of concurrent psychophysical discriminations. As shown in Figure 1, for example, discriminations are much more accurate for a task given a high proportion of attention than for a concurrent task given a low proportion of attention. The effect of attention does not seem to be "winner-take-all," as might be predicted if a unitary discrimination mechanism were always directed toward the task with higher priority. For example, discrimination is better with $100 \%$ of attention than with only $80 \%$, even though each is the condition with higher priority. Such graded effects suggest that attention is shared between tasks approximately in accordance with the instructed proportions. By itself, however, the graded effect does not indicate whether the sharing occurs within a trial or by a probability mixture across trials.

The present experiments were designed to investigate the adequacy of the sample-size model (Luce \& Green, 1978) to account for the effects of attention on psychophysical discrimination, and the results are fit adequately by a version of the model. For one thing, the model is quantitatively consistent with the performance changes observed as attention is allocated in various proportions to two concurrent discriminations, and Experiment 1 demonstrated this consistency in a new paradigm (varied allocation) and over a wider range of attentional manipulation than used previously. In addition, Experiment 2 showed that attention and physical stimulus variability have additive effects on total Thurstonian variance (Figure 4), consistent with the sample-size model. Neither of these findings confirms the model, of course, since other models may make the same predictions, or more precise data might have ruled out the present model. Nonetheless, the model must be regarded as an increasingly plausible candidate to the extent that new data are, at least to a reasonable approximation, compatible with its predictions.

In fact, the additivity of the effects of stimulus variance and attention on total Thurstonian variance seems to have fairly direct implications for Thurstonian models of attention. It is generally assumed that variance in Thurstonian models derives from three independent and additive sources: true stimulus variance, variance due to noise in the sensory system, and variance in decision criteria. The manipulation of stimulus variance influences the first of these by definition, and the present results suggest that it also affects the last. In view of this, the fact that it has an additive effect with attention suggests that attention influences the second component: variability of sensory coding. If, instead, attention influenced a process also influenced by stimulus variance, nonadditive (e.g., multiplicative) effects would seem more likely a priori than additive ones.

The quantitative account of attentional effects offered by the sample-size model is consistent with various theoretical viewpoints that describe attention as processing energy or resources (e.g., Jonides, 1983; Kahneman, 1973; Navon \& Gopher, 1979; Norman \& Bobrow, 1975; Shaw, 1978). The observations of the sample-size model can be identified with resource units in the strictest sense, since these observations satisfy the five properties of resource theories outlined by Navon (1985): (1) they have an aggregate nature, such that any number can be allocated to a certain discrimination; (2) they are exclusive (i.e., each observation is used for a single discrimination); (3) they can be distributed among discriminations, with 
different observations assigned to different tasks; (4) they control efficiency (i.e., discrimination accuracy); and (5) they are scarce (i.e., limited in total number).

Although the acceptable quantitative fit of the samplesize model to the attentional tradeoff provides a useful extension of Thurstonian models to a new experimental variable, this extension is mute regarding several issues that are commonly discussed in the literature on attention. For example, this analysis provides no way to discern the level at which attentional selection occurs: before or after low-level extraction of visual features. Attention may increase sensitivity by facilitating either extraction of visual features from the stimulus or integration of these features into an articulated perceptual representation; both possibilities would have the same result within the context of the sample-size model. Another issue not addressed by the model is whether visual attention is best conceived of as a unitary spotlight, a divided spotlight, a zoom lens, or just a generalized capacity. An all-or-none model, with a spotlight devoted to one stimulus pair on each trial, seems implausible in view of the lack of negative correlation in response accuracy across tasks and the lack of effect of blocked versus varied attentional allocation. Nonetheless, the remaining possibilities include a unitary spotlight that can switch between tasks within a trial, a divided spotlight, a zoom lens, or an abstract, generalized capacity that does not correspond well to a spotlight or lens metaphor.

Regardless of the best spatial metaphor for visual attention, the greater overall sensitivity with varied rather than blocked attentional allocation, observed in Experiment 1 , is consistent with previous suggestions that there is some inhibition of return in spatial attention (e.g., Klein, 1988; Maylor \& Hockey, 1985; Posner \& Cohen, 1984; Posner et al., 1984). Contrary to the suggestion of Spencer et al. (1988), it does not appear that attention is simply less focused with blocked than with varied allocation of attention. Rather, there seems to be less total perceptual capacity in the former task, which is consistent with the view that attending to a location leaves behind a residual inhibition that interferes with the uptake or use of further information from that location (cf. Klein, 1988).

The addition of physical variance to the stimuli (Experiment 2) produced some quite informative results with possible implications beyond the present test of the sample-size model's postulate of an effect of attention on representational variance. A straightforward incorporation of this manipulation into a Thurstonian model, described in the introduction to Experiment 2, was clearly insufficient to account for the effect of stimulus variance on total Thurstonian variance. Thus, it appears that adding physical variance to the stimuli not only added an additional source of total variance but also affected other sources of variance (e.g., sensory and/or criterion) within the signal detection system. Clearly this is a result of importance for basic psychophysical theories as well as those of attention. Furthermore, the combined effects of stimulus variance and attentional allocation can be used, at least within the context of the model developed here, to shed light on the relative magnitudes of sensory and criterion variance. The present results clearly imply that criterion variance increases with stimulus variance much faster than does sensory variance, at least over the modest range of stimulus variance examined here. They also raise the possibility that criterion variance is much larger than sensory variance even in the absence of stimulus variance, at least in this task, although the difficulty of separately estimating sensory and criterion variance in the present paradigm makes it extremely difficult to estimate the relative sizes of these two types of variance precisely.

The conclusion that criterion variance is at least as large as sensory variance is somewhat surprising, because highly successful SDT models have tended to ignore the former and attribute changes in total variance to the latter (see, e.g., Green \& Swets, 1966). Given a direct relationship between criterion and sensory variance such as the one postulated in Equation 13, however, it is not difficult to imagine that criterion variance could be camouflaged quite effectively by sensory variance, thereby presenting no obvious challenges to models in which it is ignored. For example, experimental manipulations that caused linear increases in sensory variance would also cause linear changes in total variance, simply because criterion variance would also change linearly. The linear change in total variance could easily be attributed entirely to sensory variance, with no obvious need for a second component within the total variance. Only in a case where the slope of linear increase in sensory variance can be computed on a priori grounds, as with the present experimental manipulation of stimulus variance, is there a chance to observe that total variance increases more rapidly than can be accounted for by sensory variance alone, necessitating the postulation of criterion variance as the component that accounts for the extra increase.

Both conclusions-that criterion variance is at least as large as sensory variance and that criterion but not sensory variance increases with stimulus variance-are surprising, given the analysis of Nosofsky (1983). Nosofsky had subjects identify a stimulus after one, two, three, or four independent observations of it. Not surprisingly, sensitivity increased with the number of observations. Assuming that sensory variance decreased with the number of observations but that criterion variance remained constant, Nosofsky used the rate of decrease to estimate relative sensory and criterion variance. This led to the conclusion that sensory variance was larger than criterion variance. In addition, both types of variance increased when stimulus range was increased.

A possible shortcoming with Nosofsky's method for separating sensory and criterion variance is that it assumes that repeated presentations of a stimulus influence sensory but not criterion variance. The present results suggest that, at least in the present discrimination task, criterion variance is inextricably linked with representational variance. If this were also the case in the identification task, repeated presentations of a stimulus would decrease criterion vari- 
ance indirectly, through the direct reduction in sensory variance. Then, Nosofsky's use of the rate of change in total variance to estimate sensory variance would overestimate sensory variance, because some of the change in total variance would actually be due to shifts in criterion variance. In short, Nosofsky's results can be reconciled with the present conclusions by assuming that the direct relationship between sensory and criterion variance makes it impossible to change the former without changing the latter. Alternatively, of course, the discrepant conclusions may be due to qualitative differences between the discrimination task used here and the identification task used by Nosofsky.

The direct relationship between sensory and criterion variance that we are suggesting can also be used to explain the fact that adequate fits have previously been obtained with versions of the sample-size model lacking a parameter for criterion variance (e.g., Bonnel et al., 1987; the present Experiment 1). Because it depends on sensory variance, criterion variance is influenced by attention in the same way as sensory variance is. Thus, the fact that the $\sigma_{c}^{2}$ of Equation 4 appears to be zero should not be interpreted to mean that criterion variance is zero, but should rather be interpreted to mean that there is no component of the total variance that is unaffected by attention. Again, criterion variance is difficult to isolate from sensory variance if the former responds (albeit indirectly) to any manipulation that affects the latter.

In conclusion, the results of the present experiments support the sample-size model of attentional effects in concurrent discrimination tasks. The performance tradeoff obtained by varying attentional allocation across tasks is consistent with this model under a wide range of conditions. Furthermore, evidence from some of these conditions suggests that criterion variance is an important component in Thurstonian models.

\section{REFERENCES}

AshBy, F. G., \& GotT, R. E. (1988). Decision rules in the perception and categorization of multidimensional stimuli. Journal of Experimental Psychology: Learning, Memory, \& Cognition, 14, 33-53.

BarLow, H. B. (1978). The efficiency of detecting changes of density in random dot patterns. Vision Research, 18, 637-650.

BARLow, H. B. (1980). The absolute efficiency of perceptual decisions. Philosophical Transactions of the Royal Society of London (Series B), 290, $71-82$.

Bonnel, A. M., Possamai, C. A., \& SChmitt, M. (1987). Early modulation of visual input: A study of attentional strategies. Quarterly Journal of Experimental Psychology, 39A, 757-776.

BraIDA, L. D. \& DURLACH, N. I. (1972). Intensity perception: II. Resolution in one-interval paradigms. Journal of the Acoustical Society of America, 51, 483-502.

BURgess, A., \& BARLow, H. B. (1983). The precision of numerosity discrimination in arrays of random dots. Vision Research, 23, 811-820.

Dickman, S. J., \& MEYER, D. E. (1988). Impulsivity and speed-accuracy tradeoffs in information processing. Journal of Personality \& Social Psychology, 54, 274-290.

Dorfman, D. D., \& ALF, E., JR. (1969). Maximum-likelihood estimation of parameters of signal-detection theory and determination of confidence intervals: Rating method data. Journal of Mathematical Psychology, 6, 487-496.

DowniNG, C. J. (1988). Expectancy and visual-spatial attention: Effects on perceptual quality. Journal of Experimental Psychology: Human Perception \& Performance, 14, 188-202.

DudEwICZ, E. J., \& Mishra, S. N. (1988). Modern mathematical statistics. New York: Wiley.

DURLACH, N. I., \& BraIDA, L. D. (1969). Intensity perception: I. Preliminary theory of intensity resolution. Journal of the Acoustical Society of America, 46, 372-383.

EGETH, H. [E.], \& BLECKER, D. [L.] (1971). Differential effects of familiarity on judgments of sameness and difference. Perception \& Psychophysics, 9(4), 321-326.

ERIKSEN, C. W., ST. JAMES, J. D. (1986). Visual attention within and around the field of focal attention: A zoom lens model. Perception \& Psychophysics, 40, 225-240.

GRAVETTER, F., \& LoCKHEAD, G. R. (1973). Criterial range as a frame of reference for stimulus judgment. Psychological Review, 80, 203-216.

GREEN, D. M., \& Swets, J. A. (1966). Signal detection theory and psychophysics. New York: Wiley.

Hawkins, H. L., Shafto, M. G., \& Richaroson, K. (1988). Effects of target luminance and cue validity on the latency of visual detection. Perception \& Psychophysics, 44, 484-492.

Hillyard, S. A., Munte, T. F., \& Neville, H. J. (1985). Visualspatial attention, orienting, and brain physiology. In $\mathrm{M}$. I. Posner \& O. S. M. Marin (Eds.), Attention \& performance XI: Mechanisms of attention (pp. 63-84). Hillsdale, NJ: Erlbaum.

Hoffman, J. E., \& NELSON, B. (1981). Spatial selectivity in visual search. Perception \& Psychophysics, 30, 283-290.

Hughes, H. C. (1984). Effects of flash luminance and positional expectancies on visual response latency. Perception \& Psychophysics, 36, 177-184.

INGLEBY, J. D. (1968). Decision-making processes in human perception and memory. Unpublished doctoral dissertation, University of Cambridge.

JoNIDES, J. (1980). Towards a model of the mind's eye's movement. Canadian Journal of Psychology, 34, 103-112.

JONIDES, J. (1983). Further toward a model of the mind's eye's movement. Bulletin of the Psychonomic Society, 21, 247-250.

Kahneman, D. (1973). Attention and effort. Englewood Cliffs, NJ: Prentice-Hall.

KINCHLA, R. A. (1969). An attention operating characteristic in vision (Tech. Rep. No. 29). McMaster University, Hamilton, ON, Department of Psychology.

KinCHLA, R. A. (1980). The measurement of attention. In R. S. Nickerson (Ed.), Attention and performance VIII (pp. 213-218). Hillsdale, NJ: Erlbaum.

KLEIN, R. M. (1988). Inhibitory tagging system facilitates visual search. Nature, 334, 430-431.

Kornbrot, D. E. (1980). Attention bands: Some implications for categorical judgments of loudness. Journal of Experimental Psychology: Human Perception \& Performance, 3, 92-104.

Kubovy, M., \& Healy, A. F. (1977). The decision rule in probabilistic categorization: What it is and how it is learned. Joumal of Experimental Psychology: General, 106, 427-446.

LEE, W., \& JAHNKE, M. (1964). Categorizing externally distributed stimulus samples for three continua. Journal of Experimental Psychology, 68, 376-382

Lehmann, E. L. (1986). Testing statistical hypotheses (2nd ed.). New York: Wiley

LindSAY, P. H., TAYLOR, M. M., \& Forbes, S. M. (1968). Attention and multidimensional discrimination. Perception \& Psychophysics, 4, 113-117.

LUCE, R. D. (1977), Thurstone's discriminal processes fifty years later. Psychometrika, 42, 461-489.

LuCE, R. D., \& GREEN, D. M. (1978). Two tests of a neural attention hypothesis for auditory psychophysics. Perception \& Psychophysics, 23, 363-371.

LUCE, R. D., GreEN, D. M., \& Weber, D. L. (1976). Attention bands in absolute identification. Perception \& Psychophysics, 20, 49-54.

Macmillan, N. A., Kaplan, H. L. (1985). Detection theory analysis of group data: Estimating sensitivity from average hit and false-alarm rates. Psychological Bulletin, 98, 185-199.

MAYLOR, E. A., \& HockeY, R. J. (1985). Inhibitory component of ex- 
ternally controlled covert orienting in visual space. Journal of Experimental Psychology: Human Perception \& Performance, 11, 777-787.

MiLLER, J. [O.] (1991). The flanker compatibility effect as a function of visual angle, attentional focus, visual transients, and perceptual load: A search for boundary conditions. Perception \& Psychophysics, 49, 270-288.

Moss, S. M., Myers, J. L., \& Filmore, T. (1970). Short-term recognition memory of tones. Perception \& Psychophysics, 7, 369-373.

Müller, H. J., \& Findlay, J. M. (1987). Sensitivity and criterion effects in the spatial cuing of visual attention. Perception \& Psychophysics, 42, 383-399.

NAvON, D. (1985). Attention division or attention sharing? In M. I. Posner \& O. S. M. Marin (Eds.), Attention and performance XI (pp. 133-146). Hillsdale, NJ: Erlbaum.

NAVON, D., \& GopHER, D. (1979). On the economy of the human information processing system. Psychological Review, 86, 214-255.

Norman, D. A., \& BoBRow, D. G. (1975). On data-limited and resourcelimited processes. Cognitive Psychology, 7, 44-64.

Nosofsky, R. M. (1983). Information integration and the identification of stimulus noise and criterial noise in absolute judgment. Journal of Experimental Psychology: Human Perception \& Performance, 9 , 299-309.

POSNER, M. I. (1980). Orienting of attention. Quarterly Journal of Experimental Psychology, 32, 3-25.

POSNER, M. I., \& COHEN, Y. (1984). Components of visual orienting. In H. Bouma \& D. G. Bowhuis (Eds.), Attention and performance X: Control of language processes (pp. 531-556). Hillsdale, NJ: Erlbaum.

Posner, M. I., Cohen, Y., Choate, L. S., Hockey, R. J., \& MayLOR, E. A. (1984). Sustained concentration: Passive filtering or active orienting? In S. Kornblum \& J. Requin (Eds.), Preparatory states and processes (pp. 49-65). Hillsdale, NJ: Erlbaum.

Posner, M. 1., SNyder, C., \& Davidson, B. (1980). Attention and the detection of signals. Joumal of Experimental Psychology: General, 109, 160-174.

RosENBROCK, H. H. (1960). An automatic method for finding the greatest or least value of a function. Computer Journal, 3, 175-184.

Shaw, M. L. (1978). A capacity allocation model for reaction time. Joumal of Experimental Psychology: Human Perception \& Performance, 4, 586-598.

Spencer, M. B. H., LAmbert, A. J., \& Hockey, R. (1988). The inhibitory component of orienting, alertness and sustained attention. Acta Psychologica, 69, 165-184.

SPERLING, G. (1984). A unified theory of attention and signal detection. In R. Parasuraman \& D. R. Davies (Eds.), Varieties of attention (pp. 103-182). New York: Academic Press.

SPERLiNG, G., \& Melchner, M. (1978a). The attention operating characteristic: Examples from visual search. Science, 202, 315-318.

SPERLING, G., \& MELCHNER, M. (1978b). Visual search, visual attention, and the attention operating characteristic. In J. Requin (Ed.), Attention and performance VII (pp. 675-686). Hillsdale, NJ: Erlbaum.

Stevens, S. S., \& Galanter, E. H. (1957). Ratio scales and category scales for a dozen perceptual continua. Joumal of Experimental Psychology, 54, 377-411.

Swets, J. A., \& Birusall, T. G. (1967). Deferred decision in human signal detection: A preliminary experiment. Perception \& Psychophysics, 2, 15-24.

Swets, J. A., \& Picketт, R. M. (1982). Evaluation of diagnostic systems. New York: Academic Press.

Swets, J. A., ShIPley, E. F., McKey, M. J., \& Green, D. M. (1959). Multiple observations of signals in noise. Journal of the Acoustical Society of America, 31, 514-521.

TAYLOR, M. M., LINDSAY, P. H., \& Forbes, S. M. (1967). Quantification of shared capacity processing in auditory and visual discrimination. In A. F. Sanders (Ed.), Attention and performance (Vol. 1, pp. 223-229). Amsterdam: North-Holland.

Teghtsoonian, M., \& Teghtsoonian, R. (1971). How repeatable are Stevens's power law exponents for individual subjects? Perception \& Psychophysics, 10, 147-149.

Thurstone, L. L. (1927). A law of comparative judgment. Psychological Review, 34, 273-286.
Treisman, M. (1987). Effects of the setting and adjustment of decision criteria on psychophysical performance. In E. E. Roskam \& R. Suck (Eds.), Progress in mathematical psychology (Vol. 1, pp. 253-297). Amsterdam: Elsevier.

Treisman, M., \& Faulkner, A. (1985). Can decision criteria interchange locations? Some positive evidence. Journal of Experimental Psychology: Human Perception \& Performance, 11, 187-208.

Treisman, M., \& Williams, T. C. (1984). A theory of criterion setting with an application to sequential dependencies. Psychological Review, 91, 68-111.

UMiLTÀ, C. (1988). Orienting of attention. In F. Boller \& J. Grafman (Eds.), Handbook of neuropsychology (Vol. 1, pp. 175-193). Amsterdam: Elsevier.

WICKELGREN, W. A. (1968). Unidimensional strength theory and component analysis of noise in absolute and comparative judgments. Journal of Mathematical Psychology, 5, 102-122.

\section{NOTES}

1. We will adopt the convention of referring to subjects' responses with words in quotation marks (e.g., "same"" vs. "different" responses) and to stimulus values with words in italics (e.g., same vs. different stimuli).

2. It is possible that subjects could form the internal representation of the line length difference directly, rather than by forming representations of the lengths of each line and finding the difference in those. This changes the notation somewhat, but it does not change any essential features of the model. We prefer to formulate the model in the version with internal representations of individual lines, because this version is easier to relate to the manipulation of stimulus variance, line by line, in Experiment 2.

3. This standard description of response selection in a confidence rating procedure does not address the issue of what would happen if response criteria exchanged positions (e.g., less evidence of differentness is needed to exceed the criterion for a high-confidence "different" response than to exceed the criterion for a medium-confidence "different" response). Treisman and Faulkner (1985) have presented evidence that criterion exchanges may sometimes occur, but we will not elaborate the model to include this possibility, which we assume to be rare.

4. If $\sigma_{c}^{2}$ is greater than zero, the POC predicted by this model is more concave (i.e., more sharply bowed) than a semicircle, and $d^{\prime}(i)^{2}$ is a negatively accelerated function of $P(i)$, rather than the linear function shown in Equation 6 . The deviations produced by positive criterion variance would probably not cause rejection of the model unless $\sigma_{c}^{2}$ was at least half as large as $\sigma_{s}^{2}$.

5. Furthermore, subjects' directions of gaze were later monitored through a closed-circuit TV in a concurrent brightness discrimination task with similar visual angles. Fixation-based strategies were never observed.

6. It is possible that a true negative correlation due to switching was obscured by a positive correlation of approximately the same magnitude. Such a positive correlation would be caused by trial-to-trial variation along factors that influenced performance in the same way on both tasks (e.g., arousal, preparedness, motivation). Although this possibility cannot be ruled out, it does seem implausible because of the requirement that the positive and negative sources of correlation cancel out more or less exactly.

7. Statistical comparisons between parameters estimated from pooled responses were carried out using $z$ tests, as recommended by Dorfman and Alf (1969). The parameter estimation procedure gives not only the estimated value of each parameter but also the variance of this estimate. Thus, to test the null hypothesis that the true parameter values are identical, the observed $z$ value is computed as the difference of the estimated parameter values divided by the square root of the sum of their respective variances. Similarly, $z$ values were used to test whether a single parameter was equal to a certain hypothesized value (e.g., zero). For this test, the observed $z$ value is the difference between the estimated parameter and the hypothesized value, divided by the standard deviation of the parameter estimate. Statistical tests involving average parameter estimates of individual subjects used standard measures of variability for between- or within-subjects designs, as was appropriate. 
8. For this and the other model fitting reported in this article, the Rosenbrock (1960) function minimization algorithm was used to minimize the sum across conditions of $\left[d_{e}^{\prime}\right.$ (observed) $-d_{e}^{\prime}$ (predicted) ${ }^{2}$, divided by the variance of $d_{e}^{\prime}$ (observed). For pooled estimates, $d_{e}^{\prime}$ (observed) and its variance were estimated by using the maximum-likelihood procedure of Dorfman and Alf (1969) as applied to responses pooled across subjects (cf. Macmillan \& Kaplan, 1985). For averaged estimates, the variance of $d_{e}^{\prime}$ (observed) was the square of its standard error (i.e., the variance of the individual-subject $d_{e}^{\prime}$ s divided by the sample size). Under the null hypothesis that the model is correct, the minimized sum should have approximately a chi-square distribution with degrees of freedom equal to the number of data points minus the number of free model parameters.

9. In a confidence rating task, there are actually multiple criteria, as discussed previously. Given our previous assumptions, however, distinguishing among them does not alter the equations for variance developed below, so we will ignore the multiplicity of criteria to simplify the exposition.
10. It may surprise some readers that we need to assume only a linear transformation rather than the more restrictive multiplicative scale transformation. Note, however, that the additive constant in the linear transformation cancels out in the computation of $\mu$, which is a difference in mean line lengths. Furthermore, variance measures are not affected by addition of a constant to a random variable.

11. It must be conceded, however, that the mechanisms by which this has been suggested to occur (Luce et al., 1976) seem unlikely in the present case because the range of stimulus length was rather small.

12. The equations would have a slightly simpler form if we assumed that criterion variance increased with $\sigma_{l}^{2}$ rather than $\sigma_{r}^{2}$, but in fact the two assumptions do not lead to identifiably different models for the present data.

(Manuscript received January 31, 1992 revision accepted for publication July $6,1993$. 